\title{
带有不同端炔保护基团的新型苯乙炔树枝状化合物的合成
}

\author{
许晨黄鹏程* \\ (北京航空航天大学材料科学与工程学院 北京 100191)
}

\begin{abstract}
摘要 以 1,3,5-三溴苯为原料, 通过 Sonogashira 反应，设计并合成了两种带有不同端炔保护基团的 1,3,5-取代的苯乙炔 树枝状化合物：1,3,5-三[3-(3-甲基-3-羟基-1-丁炔基)-5-(三异丙基硅乙炔基)苯基乙炔基]苯( $\mathbf{B}_{1}$ )和 1,3,5-三[3-(三甲基硅乙 炔基)-5-(三异丙基硅乙炔基)苯基乙炔基]苯 $\left(\mathbf{B}_{2}\right.$ ), 并对合成路线的选择进行了比较和讨论. 用 ${ }^{1} \mathrm{H} \mathrm{NMR},{ }^{13} \mathrm{C} N \mathrm{NMR}$, 质谱, 元素分析等表征手段确认了中间体及最终产物的结构. 这两种苯乙炔树枝状化合物各自带有两类不同的周边端炔保护 基团, 可根据其脱保护条件的不同引入不同的周边功能基团.

关键词 苯乙炔树枝状化合物; Sonogashira 反应; 1,3,5-三[3-(3-甲基-3-羟基-1-丁炔基)-5-(三异丙基硅乙炔基)苯基乙炔 基]苯; 1,3,5-三[3-(三甲基硅乙炔基)-5-(三异丙基硅乙炔基)苯基乙炔基]苯
\end{abstract}

\section{Synthesis of Novel Phenylacetylene Dendrimers Containing Different Protective Groups for Their Terminal Acetylenes}

\author{
$\mathrm{Xu}$, Chen Huang, Pengcheng* \\ (College of Material Science and Technology, Beihang University, Beijing 100191)
}

\begin{abstract}
Using Sonogashira coupling reaction, two kinds of 1,3,5-substituted phenylacetylene dendrimers: 1,3,5-tris[3-(3hydroxy-3-methyl-1-butynyl)-5-(triisopropylsilylethynyl)phenylethynyl]benzene $\left(\mathbf{B}_{1}\right)$ and 1,3,5-tris[3-(trimethylsilylethynyl)5-(triisopropylsilylethynyl)phenylethynyl]benzene $\left(\mathbf{B}_{2}\right)$ were synthetized and the synthetic routes were compared and discussed. The structures of the target compounds and intermediates were confirmed by ${ }^{1} \mathrm{H} N M R,{ }^{13} \mathrm{C}$ NMR, EI-MS, HRMS techniques and element analysis. In phenylacetylene dendrimers $\mathbf{B}_{\mathbf{1}}$ and $\mathbf{B}_{\mathbf{2}}$, the six terminal acetylene groups on their periphery were protected by two different kinds of groups, respectively. Based on the different deprotection conditions, two different kinds of functional groups could be introduced to $\mathbf{B}_{1}$ and $\mathbf{B}_{2}$, respectively.

Keywords phenylacetylene dendrimer; Sonogashira reaction; 1,3,5-tris[3-(3-hydroxy-3-methyl-1-butynyl)-5-(triisopropylsilylethynyl)phenylethynyl]benzene; 1,3,5-tris[3-(trimethylsilylethynyl)-5-(triisopropylsilylethynyl)phenylethynyl]benzene
\end{abstract}

近年来出现的树枝状化合物(也称为齐树高分子， 英文称 dendrimer)具有规整、精确对称的树枝状结构单 元，从分子中心向三维空间逐阶排列，通过支化基元逐 步重复的收玫或发散反应得到. 分子体积、形状及功能 团都可以精确控制, 典型的分子形状是球状. 由于其结 构上的特殊性，比线型的高分子有较大的溶解度, 较小 的粘度和优异的包埋小分子的性能, 在作为特种功能涂 料、交联剂、耦合剂、固化剂、密封剂、特殊的光、电、 磁性能电子器件中的应用前景引起人们极大关注 ${ }^{[1 \sim 3]}$. 其中, 苯乙炔树枝状化合物(phenylacetylene dendrimer) 作为其中具有代表性的功能性的树枝状化合物, 一直是 研究者关注的热点. 其规整刚性的共轭支化结构, 众多 的周边基团使得这类化合物在诸多领域具有潜在的应

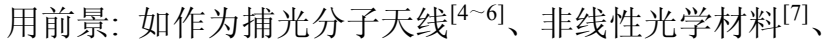
有机发光二极管材料 ${ }^{[8,9]}$ 、纳米传感器、光学纳米探针 ${ }^{[10]}$ 以及用来改性富勒烯和碳纳米管 ${ }^{[11]}$ 等. 1993 年徐志傅和 Moore ${ }^{[12]}$ 报道了至今为止人工合成的体积最大的、结构 最精致有序的四阶苯乙炔树枝状大分子，其分子直径达 到 $12.5 \mathrm{~nm}$, 被评为 1993 年世界十大科技成就之一. Adhikari 等 ${ }^{[13]}$ 报道了以咔唑为取代基团的一阶苯乙炔树 枝状化合物，作为有机发光二极管材料可发出几乎完美 的白光，由其组成的发光二极管最高亮度可达 450 $\mathrm{cd} / \mathrm{m}^{2}$, 在 $11 \mathrm{~V}$ 电压下最大效率可达到 $0.11 \mathrm{~cd} / \mathrm{A}$, 是一

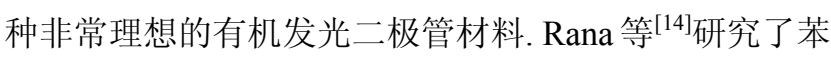
乙炔树枝状化合物的稳态光谱性质, 从理论上证明了苯 乙炔树枝状大分子作为捕光材料具有明显的优势.

*E-mail: huangpc@buaa.edu.cn

Received November 15, 2012; revised December 14, 2012; published online December 18, 2012. 
迄今为止，文献[15 17]合成的 1,3,5-取代的一阶 苯乙炔树枝状化合物大多是高度对称、周边基团完全相 同的分子，由于合成上的困难，末端带有不同保护基团 的苯乙炔树枝状化合物尚不多见. 以一阶苯乙炔树枝状 化合物 $\mathbf{A}^{[15]}$ 为例(图 1), 其周边带有 6 个完全相同的端炔 保护基团, 在脱去这些保护基团进一步发生反应时, 由 于这些保护基团完全相同, 脱去的位置和数量很难控 制，一般情况下，只能将这 6 个保护基团同时脱去. 而 得到的 6 个端炔氢在发生反应时，也很难控制发生反应 的位置和数量, 只能是 6 个端炔氢同时发生反应, 引入 6 个相同的官能团. 而在一阶苯乙炔树枝状化合物 $\mathbf{B}$ (图 1)中, 由于其分别带有两类不同的周边端炔保护基团, 可以利用脱保护反应条件的不同, 有选择性地先脱去其 中一类保护基团(如 $\mathbf{B}_{1}$ 中的三异丙基硅基， $\mathbf{B}_{2}$ 中的三甲 基硅基), 得到的 3 个端炔氢可以和一种试剂反应引入 一类官能团, 再脱去另一类保护基团(如 $\mathbf{B}_{1}$ 中的 2-羟基2-丙基, $\mathbf{B}_{2}$ 中的三异丙基硅基), 得到的 3 个端炔氢可以 和另一类试剂反应引入另一类官能团, 从而得到带有不 同种类周边官能团的苯乙炔树枝状化合物, 扩展了苯乙 炔树枝状化合物的合成范围, 对苯乙炔树枝状化合物的 功能化的分子设计和制备具有重要的理论和实际意义.

本文合成了两种带有不同周边端炔保护基团的一 阶苯乙炔树枝状化合物 $\mathbf{B}_{1}$ 和 $\mathbf{B}_{2}$, 并对合成路线的选择 进行了比较和讨论.

\section{1 结果与讨论}

\section{1 苯乙炔树枝状化合物 $B_{1}$ 的合成}

利用芳卤化合物和炔氢间的 Sonogashira 偶联反应, 按 Scheme 1 给出的合成路线, 合成了 $\mathbf{B}_{1}$. 在 Sonogashira 偶联反应中, 芳碘的活性要大于芳澳的活性 ${ }^{[18]}$, 前者可以在室温下进行, 而后者通常要在 $60{ }^{\circ} \mathrm{C}$ 以上的 温度下进行. 利用两者反应活性的差异, 可以在芳溴存 在的条件下，只发生芳碘的 Sonogashira 偶联反应. Sonogashira 偶联反应伴随的一个主要的副反应是炔氢 间的自偶联反应 ${ }^{[19]}$, 利用芳碘化合物进行 Sonoga- shira 偶联反应的另一个优点是反应可以在较低的温度下进 行, 从而可以有效抑制炔氢的自偶联反应. 在 $\mathbf{B}_{1}$ 的合成 中, 引入端炔保护基的化学试剂通常有三甲基硅炔, 三 异丙基硅炔等三烷基硅炔和 2-甲基-3-丁炔-2-醇，前者 的价格十分昂贵，而 2-甲基-3-丁炔-2-醇的价格却十分 便宜. 但在 Sonogashira 偶联反应中, 2-甲基-3-丁炔- 2醇的使用并不普遍，原因是在经过 2-甲基-3-丁炔-2-醇 引入端炔基后，其随后的脱去 2-羟基-2-丙基制备端炔 氢的反应条件十分苛刻, 通常需要强碱、高温以及长时 间 ${ }^{[20,21]}$ 的反应，因而极易引起其它副反应发生，导致
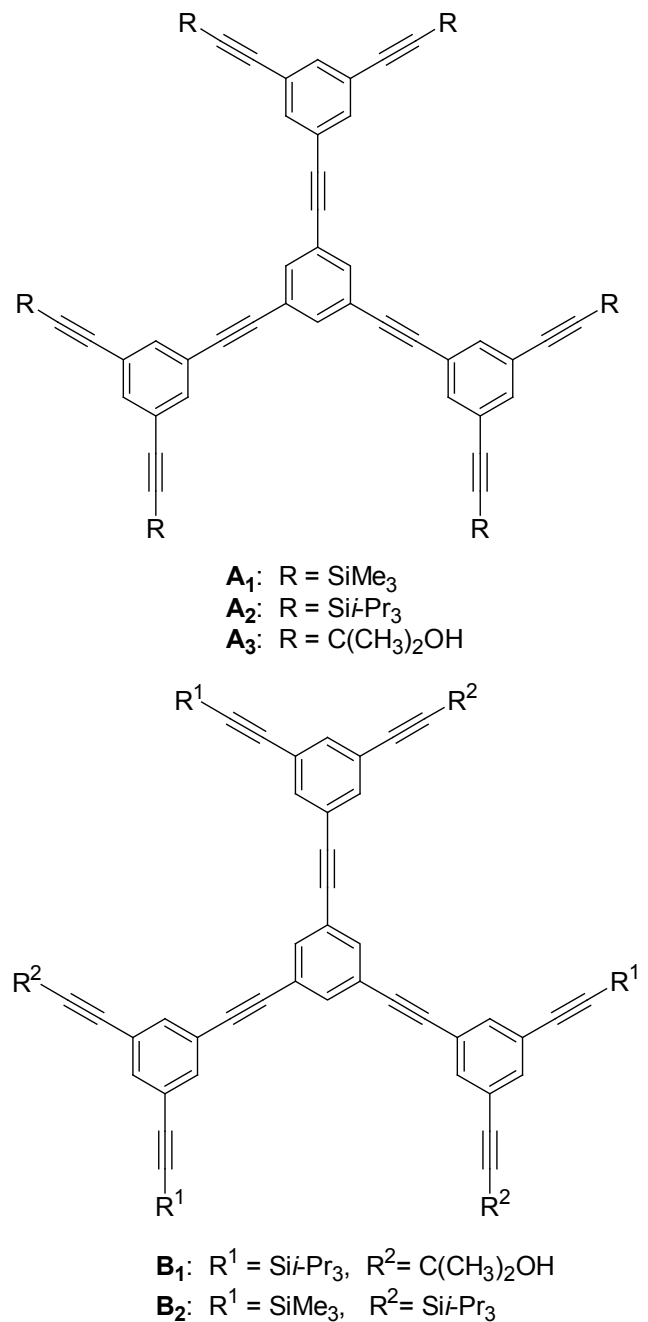

图 1 带有相同或不同保护基团的苯乙炔树枝状化合物

Figure 1 Phenylacetylene dendrimers bearing same or different peripheral protective groups

产物产率较低，分离困难，大大限制了其在合成端炔氢 中的应用. 但最近有文献[22]报道在四丁基氢氧化铵及 适量的甲醇存在条件下，脱去 2-羟基-2-丙基制备端炔 氢的反应可以在较低的温度下 $\left(65{ }^{\circ} \mathrm{C}\right)$ 经过几分钟或几 十分钟即可完成. 因此在 $\mathbf{B}_{1}$ 中，其不同的炔氢保护基团 选用了三异丙基硅基和来源于 2-甲基-3-丁炔- 2-醇的 2羟基-2-丙基.

在 Scheme 1 中化合物 $\mathbf{3}$, 也可以用 1,3,5-三溴苯(1) 和等物质的量的三甲基硅炔反应制备. 但是由于 $\mathrm{Br}$ 和 三甲基硅炔基的极性很相近, 在用色谱柱对产物进行分 离时，很难将目标产物 3、未反应的原料 1 及二取代和 三取代的副产物分开，导致最终产物的得率很低 ${ }^{[23]}$. 因 此在 Scheme 1 中, 将 $\mathbf{1}$ 中的一个溴用碘取代, 利用芳碘 和芳溴活性的差异，在较低的温度下，使三甲基硅炔只 和一个芳碘发生 Sonogashira 偶联反应，而其余的芳溴 不参加反应，从而避免了二取代和三取代副产物的生 

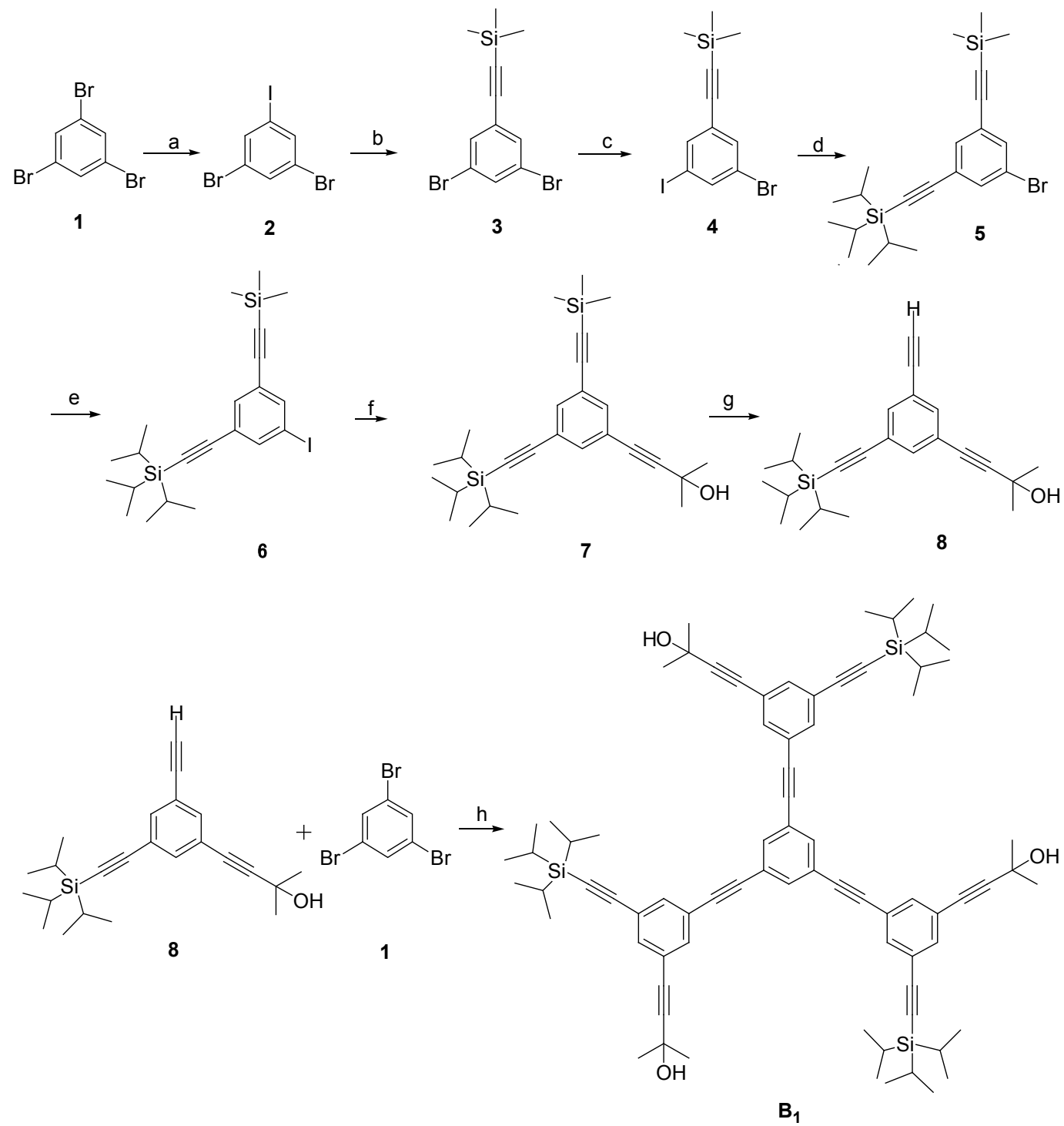

Reagents and conditions: (a) 1) 1 equiv. $n$-BuLi, $-78{ }^{\circ} \mathrm{C}, \mathrm{Et}_{2} \mathrm{O}, 2 \mathrm{~h}$; 2) $\mathrm{I}_{2} / \mathrm{Et}_{2} \mathrm{O}$ (83\%). (b) $\mathrm{PdCl}_{2}\left(\mathrm{PPh}_{3}\right)_{2}$, Cul, Trimethylsilylacetylene, $\mathrm{NEt}_{3}$, THF, $0{ }^{\circ} \mathrm{C}, 30 \mathrm{~min}(95 \%)$. (c) 1) 1 equiv. $n$-BuLi, $\left.-78{ }^{\circ} \mathrm{C}, \mathrm{Et}_{2} \mathrm{O}, 2 \mathrm{~h} ; 2\right) \mathrm{ICH}_{2} \mathrm{CH}_{2} \mathrm{l} / \mathrm{Et}_{2} \mathrm{O}$ (93\%). (d) $\mathrm{PdCl}_{2}\left(\mathrm{PPh}_{3}\right)_{2}$, Cul, Triisopropylsilylacetylene, $\mathrm{NEt}_{3}, 20^{\circ} \mathrm{C}, 3 \mathrm{~d}(94 \%)$. (e) 1 equiv. $n$-BuLi, $-78{ }^{\circ} \mathrm{C}, \mathrm{Et}_{2} \mathrm{O}$, $2 \mathrm{~h}$; 2) $\mathrm{ICH}_{2} \mathrm{CH}_{2} \mathrm{l} / \mathrm{Et}_{2} \mathrm{O}$ (92\%). (f) $\mathrm{PdCl}_{2}\left(\mathrm{PPh}_{3}\right)_{2}, \mathrm{Cul}_{2} \mathrm{PPh}_{3}, 2$-methyl-3-butyn-2-ol, $\mathrm{NEt}_{3}, 20^{\circ} \mathrm{C}, 3 \mathrm{~d}(98 \%)$. (g) $\mathrm{CH}_{3} \mathrm{OH}, \mathrm{CH}_{2} \mathrm{Cl}_{2}, \mathrm{KOH}, 20^{\circ} \mathrm{C}, 3 \mathrm{~h}(97 \%)$. (h) $\mathrm{PdCl}_{2}\left(\mathrm{PPh}_{3}\right)_{2}, \mathrm{Cul}, \mathrm{PPh}_{3}, \mathrm{NEt}_{3}, 65^{\circ} \mathrm{C}, 3 \mathrm{~d}(89 \%)$.

\section{Scheme 1}

成, 使产物易于分离, 而且该反应产率很高. 基于同样 的理由, 5 的合成也是先用碘取代一个溴再与三异丙基 硅炔反应来制备.

在 Scheme 1 中, 化合物 7 带有 3 个不同端炔保护基, 其引入的顺序为三甲基硅基, 三异丙基硅基和 2-羟基2-丙基. 之所以采用这样的顺序是因为如果过早地引入 2-差基-2-丙基, 其带有的羟基会和碘代反应中的正丁基 锂反应, 干扰了碘代反应的进行, 所以 2-羟基-2-丙基要 最后引入. 至于先引入三甲基硅基再引入三异丙基硅基 是因这一顺序可以获得较高的得率 ${ }^{[24]}$.

最后, 以 $1,3,5$-三澳苯为核, 用化合物 8 与其发生 Sonogashira 偶联反应，可以高得率 $(89 \%)$ 地合成 $\mathbf{B}_{\mathbf{1}}$.

\section{2 苯乙炔树枝状化合物 $B_{2}$ 的合成}

$\mathbf{B}_{1}$ 的六个周边炔基带有两种不同的保护基团，可 以利用它们脱保护条件的不同，逐次脱去一种保护基 团，在 $\mathbf{B}_{1}$ 中引入不同的功能基团. 但是 $\mathbf{B}_{1}$ 也有一个不 足, 就是带有的 2-羟基-2-丙基中的羟基对某些有质子 参与的反应会起干扰作用，因而使其在后续反应的使用 中受到限制. 为此, 我们设计合成了苯乙炔树枝状化合 物 $\mathbf{B}_{2}$, 它的 6 个周边端炔的保护基团分别为三甲基硅基 和三异丙基硅基, 对 $\mathbf{B}_{2}$ 的后续反应一般不会发生干扰.

我们设计了两条路线 Scheme 2 和 Scheme 3 来合成

$\mathbf{B}_{2}$, 其主要的不同在于 Scheme 2 中采用 1,3,5-三乙炔基 
苯为核, 用化合物 6 与其进行 Sonogashira 反应来合成 $\mathbf{B}_{2}$. 而在 Scheme 3 中, 采用 1,3,5-三溴苯为核, 用化合 物 11 与其进行 Sonogashira 反应来合成 $\mathbf{B}_{2} .1,3,5$-三乙炔 基苯本身就有三个端炔氢, 化学性质活泼, 使其在 Sonogashira 偶联反应中更容易发生自偶联的副反应, 使产物体系极为复杂, 产物分离困难, 产率降低 ${ }^{[25]}$. 而 Scheme 3 的路线可以避免这一点. 就这一意义而言, Scheme 3 的合成路线要优于 Scheme 2. 但在 Scheme 3 中, 需要从化合物 7 中脱去 2-羟基-2-丙基制备化合物 11. 虽然有文献[26]报道在类似的脱保护反应中, 在绝 对无水的条件下, 可在脱去 2-羊基-2-丙基的同时, 保证
三甲基硅基不被脱去．但在我们的实验中，按文献[26] 的方法从 7 制备 11 时, 无论怎样控制反应条件, 都无法 保证在脱去 2-羟基-2-丙基的同时, 7 中的三甲基硅基不 被脱去, 且目标产物 11 和副产物极性很相近, 导致分离 困难, 从而使 11 的产率很低. 因此, 在本论文中, 是按 Scheme 2 的路线来合成 $\mathbf{B}_{2}$, 其最后一步的得率也可以 达到 $51 \%$.

\section{2 结论}

利用芳碘和芳溴在发生 Sonogashira 偶联反应的条 件不同，将化合物 $\mathbf{1}$ 和 $\mathbf{3}$ 中的溴用等物质的量的碘取代<smiles>C#Cc1cc(C#C)cc(C#C)c1</smiles><smiles>C#Cc1cc(C#C)cc(C#C)c1</smiles>

Reagents and conditions: (a) $\mathrm{PdCl}_{2}\left(\mathrm{PPh}_{3}\right)_{2}, \mathrm{Cul}, \mathrm{PPh}_{3}, \mathrm{NEt}_{3}, 2$-Methyl-3-butyn-2-ol, $65^{\circ} \mathrm{C}, 2 \mathrm{~d}(89 \%)$. (b) $\mathrm{Bu}_{4} \mathrm{NOH}$, $\mathrm{CH}_{3} \mathrm{OH}$, Toluene, $75^{\circ} \mathrm{C}(90 \%)$. (c) $\mathrm{PdCl}_{2}\left(\mathrm{PPh}_{3}\right)_{2}, \mathrm{Cul} \mathrm{PPh}_{3}$, Piperidine, $20^{\circ} \mathrm{C}, 4 \mathrm{~d}(51 \%)$

Scheme 2

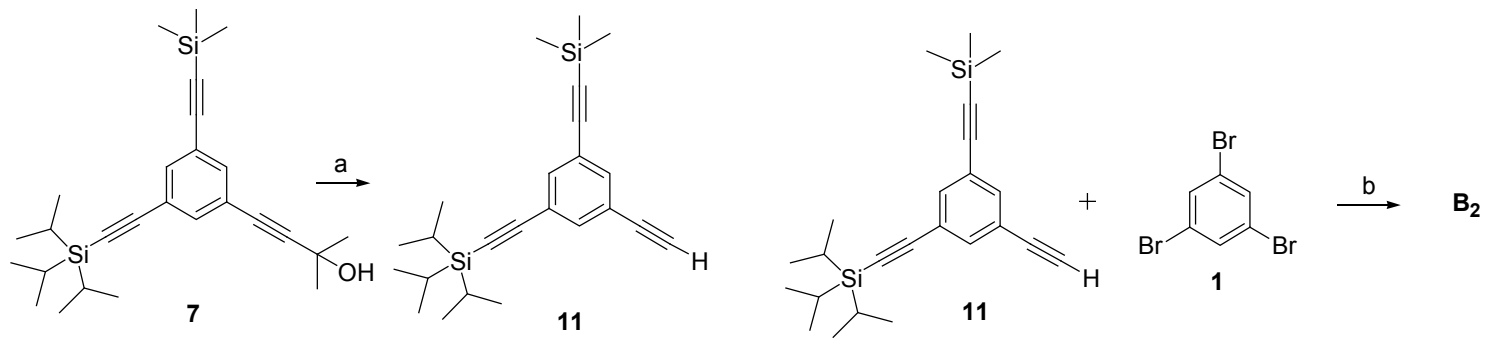

Reagents and conditions: (a) toluene, $\mathrm{KOH}, \mathrm{Ar}, 120^{\circ} \mathrm{C}$. (b) $\mathrm{PdCl}_{2}\left(\mathrm{PPh}_{3}\right)_{2}, \mathrm{Cul}, \mathrm{PPh}_{3}, \mathrm{NEt}_{3}, 65^{\circ} \mathrm{C}$

Scheme 3 
进而合成化合物 3 和 $\mathbf{5}$, 可以避免多重取代副产物的生 成, 使产物易于分离, 提高了 $\mathbf{3}$ 和 $\mathbf{5}$ 的得率. 化合物 7 带有的 3 个不同端炔保护基, 其引入的顺序应为三甲基 硅基，三异丙基硅基和 2-垟基-2-丙基.

按照 Scheme 1 和 Scheme 2 的合成路线, 可以高产 率地得到带有两类不同端炔保护基团的一阶苯乙炔树 枝状化合物：1,3,5-三[3-(3-甲基-3-羟基-1-丁炔基)-5-(三 异丙基硅乙炔基)苯基乙炔基]苯( $\mathbf{B}_{1}$ )和 1,3,5-三[3-(三甲 基硅乙炔基)-5-(三异丙基硅乙炔基)苯基乙炔基苯]苯 $\left(\mathbf{B}_{2}\right)$. 利用保护基团的脱保护条件的不同, 可以逐次脱 去各类保护基团，从而在同一苯乙炔树枝状化合物中引 入不同功能的周边基团, 扩展了苯乙炔树枝状化合物的 合成范围, 为苯乙炔树枝状化合物的分子设计和功能化 应用提供了新的合成途径.

\section{3 实验部分}

\section{1 仪器与试剂}

熔点测定仪采用巩义市予华仪器有限责任公司生 产的 XT 5 型熔点仪测定; 核磁共振氢谱 ${ }^{1} \mathrm{H}$ NMR (400 $\mathrm{MHz}$ )和 ${ }^{13} \mathrm{C}$ NMR (100 MHz) 采用 Bruker AV 400 核磁共 振谱仪测定, 所用溶剂为 $\mathrm{CDCl}_{3}$, TMS 为内标, 室温下 测定; 元素分析采用 vario EL III 元素分析仪测定; EI-MS 采用 Finnigan TRACE 2000 气质联用仪测定; HRMS (ESI)采用 Bruker APEX IV FT-MS (7.0T)高分辨 质谱仪测定. $\mathrm{PdCl}_{2}\left(\mathrm{PPh}_{3}\right)_{2}$ 为 Aldrich 分析纯产品; 三甲 基硅炔、三异丙基硅炔、1,2-二碘乙烷为 Acros 分析纯 产品; 其它化学试剂和溶剂均为市售分析纯产品. 四氢 呋喃和乙醚在钠/二苯甲酮回流除水, 在惰性气体保护 下蒸馏, 现蒸现用; 哌啶和三乙胺使用前用 $\mathrm{CaH}_{2}$ 回流 除水, 常压蒸馏, 并用 $\mathrm{Ar}$ 气鼓泡除氧 $30 \mathrm{~min}$; 间三澳苯 (工业品), 使用前无水乙醇中重结晶后柱层析提纯.

\section{2 实验方法}

\subsubsection{1,3-二澳-5-碘苯(2)的合成}

向装有低温温度计、气囊、磁子的 $1000 \mathrm{~mL}$ 干燥四 口烧瓶中加入 1,3,5-三澳苯(1) (18.860 g, $59.91 \mathrm{mmol})$, 用橡胶翻口塞密闭, 对体系抽真空、通 $\mathrm{Ar}$ 气操作 4 次, 最后使得体系中充满惰性气体. 向反应瓶中注入 550 $\mathrm{mL}$ 的无水乙醚后将烧瓶置于液氮/乙醇浴中, 调节体系 温度到 $-78{ }^{\circ} \mathrm{C}$, 惰性气体保护下用注射器抽取正丁基 锂/正己烷溶液 $(25.0 \mathrm{~mL}, 60 \mathrm{mmol}$ )慢慢滴入反应体系, 期间保持体系温度在 $-78 \sim-70{ }^{\circ} \mathrm{C}$ 之间, $20 \mathrm{~min}$ 滴完， 保持 $-78 \sim-75{ }^{\circ} \mathrm{C}$ 反应 $2 \mathrm{~h}$ 生成相应的苯锂溶液. 向另 一干燥的三口瓶中加入碘(19.771 g, $77.88 \mathrm{mmol})$, 抽真 空进 $\mathrm{Ar}$ 气 4 次后注入 $340 \mathrm{~mL}$ 无水乙醚, 将形成的碘的 乙醚溶液用注射器缓慢滴加至四口烧瓶中, 滴加过程中
保持体系温度维持在一 $78 \sim-70{ }^{\circ} \mathrm{C}$ 之间，滴加完毕后 继续保持在 $-78 \sim-75{ }^{\circ} \mathrm{C}$ 反应 $2 \mathrm{~h}$, 升温搅拌过夜. 反 应生成的溶液用饱和 $\mathrm{Na}_{2} \mathrm{SO}_{3}$ 洗, 有机相用饱和 $\mathrm{NaCl}$ 水 溶液洗涤, 无水 $\mathrm{MgSO}_{4}$ 干燥. 过滤后减压蒸去溶剂. 粗 产物在无水乙醇中经过两次重结晶得到白色针状晶体 2, $17.935 \mathrm{~g}$, 产率 83\%. m.p. $123.7 \sim 124.4{ }^{\circ} \mathrm{C}$ (文献 值 $\left.{ }^{[27]} 124.4 \sim 124.7{ }^{\circ} \mathrm{C}\right) ;{ }^{1} \mathrm{H}$ NMR $\left(400 \mathrm{MHz}, \mathrm{CDCl}_{3}\right) \delta$ : 7.795 (d, $J=1.6 \mathrm{~Hz}, 2 \mathrm{H}, \mathrm{ArH}), 7.638$ (t, $J=3.2 \mathrm{~Hz}, 1 \mathrm{H}$, $\mathrm{ArH}) ;{ }^{13} \mathrm{C}$ NMR $\left(100 \mathrm{MHz}, \mathrm{CDCl}_{3}\right) \delta: 138.5,133.7,123.4$, 94.4; MS m/z (\%): $362\left(\mathrm{M}^{+}, 38\right), 235$ (13), 127 (100), 74 (82). Anal. calcd for $\mathrm{C}_{6} \mathrm{H}_{3} \mathrm{Br}_{2} \mathrm{I}$ : C 19.92, H 0.84; found C 20.04, H 0.89 .

\subsubsection{1,3-二溴-5 三甲基硅乙炔基苯(3)的合成}

向 $250 \mathrm{~mL}$ Schlenk 瓶中加入 1,3-二溴-5-碘苯 2 (10.07 g, $27.83 \mathrm{mmol})$, CuI (0.5274 g, $2.77 \mathrm{mmol})$, $\mathrm{PdCl}_{2}\left(\mathrm{PPh}_{3}\right)_{2}(780.4 \mathrm{mg}, 1.1 \mathrm{mmol})$, 体系抽真空通 $\mathrm{Ar}$ 气 四次后，向反应瓶中注入无水 THF $100 \mathrm{~mL}$, 三乙胺 30 $\mathrm{mL}$, 然后将 Schlenk 瓶置于冰浴中, 磁力摚拌下用注射 器向反应体系中注入三甲基硅炔 $(2.733 \mathrm{~g}, 27.83 \mathrm{mmol})$, 在冰浴的条件下反应, TLC 监测, $30 \mathrm{~min}$ 后原料消失, 停 止反应. 减压蒸干溶剂, 剩余物用 $200 \mathrm{~mL} \mathrm{CH} \mathrm{Cl}_{2}$ 溶解, 有机相依次以稀盐酸 $(5 \%)$ 及饱和 $\mathrm{NaCl}$ 洗涤, 无水 $\mathrm{MgSO}_{4}$ 干燥, 过滤. 减压抽干溶剂后粗产物以柱色谱提 纯(石油醚为淋洗液), 得无色油状液体 $\mathbf{3}, 8.72 \mathrm{~g}$, 产率 95\%. ${ }^{1} \mathrm{H}$ NMR (400 MHz, $\left.\mathrm{CDCl}_{3}\right) \delta: 7.606$ (t, $J=3.6 \mathrm{~Hz}$, 1H, ArH), 7.531 (d, $J=2.0 \mathrm{~Hz}, 2 \mathrm{H}, \mathrm{ArH}), 0.241$ (s, 9H); ${ }^{13} \mathrm{C}$ NMR $\left(100 \mathrm{MHz}, \mathrm{CDCl}_{3}\right) \delta: 134.2,133.4,126.5,122,5$, 101.7, 97.6, -0.3; MS $m / z(\%): 332\left(\mathrm{M}^{+}, 38\right), 317$ (100), 287 (15). Anal. calcd for $\mathrm{C}_{11} \mathrm{H}_{12} \mathrm{Br}_{2} \mathrm{Si}$ : C 39.78, H 3.64; found C 39.84, H 3.57.

\subsubsection{1-溴-3-碈-5-三甲基硅乙炔基苯(4)的合成}

合成步骤和 2 类似, $500 \mathrm{~mL}$ 四口瓶中加入 1,3-二溴5-碘苯(3) (8.03 g, $24.18 \mathrm{mmol})$, 体系 $\mathrm{Ar}$ 保护, 注入无水 乙醚 $250 \mathrm{~mL}$, 将烧瓶置于液氮/乙醇浴中, 调节体系温 度到 $-78{ }^{\circ} \mathrm{C}$, 搅拌条件下注入正丁基锂 $(10.1 \mathrm{~mL}, 24.24$ $\mathrm{mmol})$, 滴加过程中保持体系温度在 $-78 \sim-70{ }^{\circ} \mathrm{C}$ 之 间, 维持一 $78{ }^{\circ} \mathrm{C}$ 下反应 $2 \mathrm{~h}$ 后注入 $50 \mathrm{~mL} \mathrm{ICH}_{2} \mathrm{CH}_{2} \mathrm{I}(10$ $\mathrm{g}, 35.48 \mathrm{mmol})$ 的乙醚溶液, 维持 $-78{ }^{\circ} \mathrm{C}$ 反应 $2 \mathrm{~h}$ 后停 止反应, 缓慢升至室温. 反应液用去离子水洗 3 次, 有 机相用无水 $\mathrm{MgSO}_{4}$ 干燥, 过滤. 减压抽干溶剂后粗产物 以柱色谱提纯(石油醚为淋洗液), 过量的 $\mathrm{ICH}_{2} \mathrm{CH}_{2} \mathrm{I}$ 在 $70{ }^{\circ} \mathrm{C}$ 浴下用油原抽干, 得无色油状液体 4, $8.52 \mathrm{~g}$, 产率 93\%. ${ }^{1} \mathrm{H}$ NMR (400 MHz, $\left.\mathrm{CDCl}_{3}\right) \delta: 7.80$ (t, $J=3.2 \mathrm{~Hz}$, $1 \mathrm{H}, \operatorname{ArH}), 7.735$ (t, $J=2.8 \mathrm{~Hz}, 1 \mathrm{H}, \operatorname{ArH}), 7.665$ (t, $J=3.2$ $\mathrm{Hz}, 1 \mathrm{H}, \mathrm{ArH}), 0.238$ (s, 9H); ${ }^{13} \mathrm{C}$ NMR (100 MHz, $\mathrm{CDCl}_{3}$ ) 
$\delta: 139.7,139.1,133.9,126.6,122.4,101.5,97.6,93.6$, -0.3; MS m/z (\%): $380\left(\mathrm{M}^{+}, 19\right), 365$ (78), 253 (17), 79 (100). Anal. calcd for $\mathrm{C}_{11} \mathrm{H}_{12}$ BrISi: C 34.85, H 3.19; found C 34.86, H 3.16 .

3.2 .4 1-溴-3-三甲基硅乙炔基-5-三异丙基硅乙炔基 苯 $(5)$ 的合成

向 $100 \mathrm{~mL}$ Schlenk 瓶中加入 1-溴-3-碘-5-三甲基硅 乙炔基苯(4) (4.01 g, $10.56 \mathrm{mmol}), \mathrm{CuI}$ (0.056 g, 0.294 $\mathrm{mmol}), \mathrm{PdCl}_{2}\left(\mathrm{PPh}_{3}\right)_{2}(0.056 \mathrm{~g}, 0.0798 \mathrm{mmol})$, 体系抽真 空, 通 $\mathrm{Ar}$ 气 4 次后, 用注射器将已鼓泡除氧 $30 \mathrm{~min}$ 的 三乙胺 $36 \mathrm{~mL}$ 注入反应瓶中, 然后用注射器向体系滴 入三异丙基硅基乙炔(1.992 g, $10.56 \mathrm{mmol})$, 室温摚拌避 光反应, TLC 监测, 反应 $3 \mathrm{~d}$ 后, 原料点消失, 停止反应.

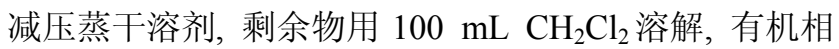
依次以稀盐酸 $(5 \%)$ 及饱和 $\mathrm{NaCl}$ 洗涤, 无水 $\mathrm{MgSO}_{4}$ 干燥, 过滤. 减压抽干溶剂后粗产物以柱色谱提纯(石油醚为 淋洗液), 得无色油状液体 5, $4.29 \mathrm{~g}$, 产率 94\%. ${ }^{1} \mathrm{H} \mathrm{NMR}$ $\left(400 \mathrm{MHz}, \mathrm{CDCl}_{3}\right) \delta: 7.537$ (t, $\left.J=3.6 \mathrm{~Hz}, 2 \mathrm{H}, \mathrm{ArH}\right), 7.482$ (t, $J=2.8 \mathrm{~Hz}, 1 \mathrm{H}, \mathrm{ArH}), 1.116(\mathrm{~s}, 21 \mathrm{H}), 0.240(\mathrm{~s}, 9 \mathrm{H}) ;{ }^{13} \mathrm{C}$ NMR $\left(100 \mathrm{MHz}, \mathrm{CDCl}_{3}\right) \delta: 134.6,134.4,133.9,125.4$, 125.0, 121.6, 104.4, 102.5, 96.6, 93.2, 18.6, 11.2, -0.3; MS m/z (\%): $435\left(\mathrm{M}^{+}, 5\right), 419$ (6), 389 (65), 319 (98), 43 (100). Anal. calcd for $\mathrm{C}_{22} \mathrm{H}_{33} \mathrm{BrSi}_{2}$ : C 60.94, H 7.67; found C 60.88, H 7.57.

3.2 .5 1-碘-3-三甲基硅乙炔基-5-三异丙基硅乙炔基 苯 $(6)$ 的合成

合成方法与合成 4 的方法一致，1-溴-3-三甲基硅乙 炔基-5-三异丙基硅乙炔基苯(5) (3.79 g, $8.74 \mathrm{mmol}$ ), 正 丁基锂(3.86 mL, $9.26 \mathrm{mmol}), \mathrm{ICH}_{2} \mathrm{CH}_{2} \mathrm{I}(3.95 \mathrm{~g}, 14.01$ $\mathrm{mmol}$ ), 反应液用去离子水洗 3 次, 有机相用无水 $\mathrm{MgSO}_{4}$ 干燥, 过滤, 减压抽干溶剂后粗产物以柱色谱提 纯(石油醚为淋洗液), 过量的 $\mathrm{ICH}_{2} \mathrm{CH}_{2} \mathrm{I}$ 在 $70{ }^{\circ} \mathrm{C}$ 下用油 泵抽干, 得淡黄色油状液体 6, $3.87 \mathrm{~g}$, 产率 $92 \%$. ${ }^{1} \mathrm{H}$ NMR (400 MHz, $\left.\mathrm{CDCl}_{3}\right) \delta: 7.538(\mathrm{~d}, J=1.6 \mathrm{~Hz}, 2 \mathrm{H}$, ArH), 7.483 (t, $J=2.8 \mathrm{~Hz}, 1 \mathrm{H}, \mathrm{ArH}), 1.115$ (s, 21H), 0.240 $(\mathrm{s}, 9 \mathrm{H}) ;{ }^{13} \mathrm{C}$ NMR $\left(100 \mathrm{MHz}, \mathrm{CDCl}_{3}\right) \delta: 140.3,140.1$, 134.5, 125.4, 125.0, 104.2, 102.3, 96.6, 93.2, 92.9, 18.6, 11.2, -0.2; MS m/z (\%): $480\left(\mathrm{M}^{+}, 9\right), 465$ (7), 437 (82), 183 (100). Anal. calcd for $\mathrm{C}_{22} \mathrm{H}_{33} \mathrm{ISi}_{2}$ : C 54.98, H 6.92; found C 55.03, H 6.84 .

3.2.6 1-三甲基硅乙炔基-3-(3-甲基-3-羟基-1-丁炔 基)-5-三异丙基硅乙炔基苯(7)的合成

向 $100 \mathrm{~mL}$ Schlenk 瓶中加入 1-碘-3-三甲基硅乙炔 基-5-三异丙基硅乙炔基苯(6) (1.501 g, $3.12 \mathrm{mmol}), \mathrm{CuI}$ (0.023 g, $0.12 \mathrm{mmol}), \mathrm{PdCl}_{2}\left(\mathrm{PPh}_{3}\right)_{2}(0.042 \mathrm{~g}, 0.06 \mathrm{mmol})$,
$\mathrm{PPh}_{3}(0.063 \mathrm{~g}, 0.24 \mathrm{mmol})$, 体系抽真空, 通 $\mathrm{Ar}$ 气 4 次后, 用注射器将已鼓泡除氧 $30 \mathrm{~min}$ 的三乙胺 $45 \mathrm{~mL}$ 注入反 应瓶中, 然后用注射器向体系滴入已鼓泡除氧 $30 \mathrm{~min}$ 的 2-甲基-3-丁炔-2-醇(0.265 g, $3.15 \mathrm{mmol})$, 室温摚拌避光 反应, TLC 监测, 反应 $2 \mathrm{~d}$ 后, 产物点不再发生变化, 停 止反应. 减压蒸干溶剂, 剩余物用 $100 \mathrm{~mL} \mathrm{CH} \mathrm{Cl}_{2}$ 溶解, 有机相依次以稀盐酸 $(5 \%)$ 及饱和 $\mathrm{NaCl}$ 洗涤, 无水 $\mathrm{MgSO}_{4}$ 干燥, 过滤. 减压抽干溶剂后粗产物以柱色谱提 纯(石油醚为淋洗液), 得浅黄色油状液体 7, $1.337 \mathrm{~g}$, 产 率 98\%. ${ }^{1} \mathrm{H}$ NMR (400 MHz, $\left.\mathrm{CDCl}_{3}\right) \delta: 7.487$ (t, $J=2.8$ $\mathrm{Hz}, 1 \mathrm{H}, \mathrm{ArH}), 7.450$ (d, $J=1.6 \mathrm{~Hz}, 2 \mathrm{H}, \mathrm{ArH}), 2.041$ $2.043(\mathrm{~m}, 1 \mathrm{H}, \mathrm{OH}), 1.595\left(\mathrm{~s}, 6 \mathrm{H}, \mathrm{CH}_{3}\right), 1.115(\mathrm{~s}, 21 \mathrm{H})$, $0.236(\mathrm{~s}, 9 \mathrm{H}) ;{ }^{13} \mathrm{C}$ NMR $\left(100 \mathrm{MHz}, \mathrm{CDCl}_{3}\right) \delta: 134.8$, $134.7,134.6,124.0,123.7,123,2,105.1,103.2$, 95.6, 94.9, 92.1, 80.6, 65.5, 31.4, 18.6, 11.3, -0.2; MS m/z (\%): 437 $\left(\mathrm{M}^{+}, 6\right), 422$ (8), 393 (100). Anal. calcd for $\mathrm{C}_{27} \mathrm{H}_{40} \mathrm{Si}_{2} \mathrm{O}: \mathrm{C}$ 74.25, H 9.23; found C 74.01, H 9.35.

3.2.7 1-乙炔基-3-(3-甲基-3-着基-1-丁炔基)-5-三异 丙基硅乙炔基苯 $(8)$ 的合成

将 7 (1.220 g, $2.79 \mathrm{mmol}$ ) 溶于 $\mathrm{CH}_{2} \mathrm{Cl}_{2}(20 \mathrm{~mL})$ 及 $\mathrm{CH}_{3} \mathrm{OH}(10 \mathrm{~mL})$ 中, $\mathrm{N}_{2}$ 保护下向其中加入粉末状 $\mathrm{KOH}$ (0.196 g, $3.50 \mathrm{mmol})$, TLC 监控, 室温反应 $3 \mathrm{~h}$, 反应结 束后将混合物倒入至饱和 $\mathrm{NaCl}(50 \mathrm{~mL})$ 溶液中, 分出有 机相, 无水 $\mathrm{MgSO}_{4}$ 干燥, 过滤. 减压抽干溶剂后粗产物 以柱色谱提纯(石油醚为淋洗液), 得淡黄色油状液体 $\mathbf{8}$, $0.988 \mathrm{~g}$, 产率 97\%. ${ }^{1} \mathrm{H} \mathrm{NMR}\left(400 \mathrm{MHz}, \mathrm{CDCl}_{3}\right) \delta: 7.510$ (t, $J=2.8 \mathrm{~Hz}, 1 \mathrm{H}, \mathrm{ArH}), 7.488$ (t, $J=3.2 \mathrm{~Hz}, 1 \mathrm{H}, \mathrm{ArH})$, 7.460 (t, $J=2.8 \mathrm{~Hz}, 1 \mathrm{H}, \mathrm{ArH}), 3.079$ (s, 1H), 2.174 (s, 1H, $\mathrm{OH}), 1.603\left(\mathrm{~s}, 6 \mathrm{H}, \mathrm{CH}_{3}\right), 1.120(\mathrm{~s}, 21 \mathrm{H}) ;{ }^{13} \mathrm{C}$ NMR $(100$ $\left.\mathrm{MHz}, \mathrm{CDCl}_{3}\right) \delta: 135.1,135.0,134.7,124.2,123.4,122.7$, 105.0, 95.1, 92.4, 81.9, 80.5, 78.3, 66.5, 31.4, 18.6, 11.3; MS m/z (\%): $364\left(\mathrm{M}^{+}, 8\right), 349$ (5), 321 (100). Anal. calcd for $\mathrm{C}_{24} \mathrm{H}_{32} \mathrm{SiO}$ : C 79.06, H 8.85; found C 78.97, H 8.90.

\section{2 .8 苯乙炔树枝状化合物 B1 合成}

向 $100 \mathrm{~mL}$ Schlenk 瓶中加入 1-乙炔基-3-(3-甲基-3羟基-1-丁炔基)-5-三异丙基硅乙炔基苯(8) (0.881 g, 2.4 $\mathrm{mmol}), 1,3,5$-三溴苯(1) (0.252 g, $0.8 \mathrm{mmol}), \mathrm{CuI}(0.018 \mathrm{~g}$, $0.096 \mathrm{mmol}), \mathrm{PdCl}_{2}\left(\mathrm{PPh}_{3}\right)_{2}(0.035 \mathrm{~g}, 0.05 \mathrm{mmol}), \mathrm{PPh}_{3}$ $(0.039 \mathrm{~g}, 0.15 \mathrm{mmol})$ 体系抽真空, 通 $\mathrm{Ar}$ 气 4 次后, 用注 射器将已鼓泡除氧 $30 \mathrm{~min}$ 的三乙胺 $45 \mathrm{~mL}$ 注入反应瓶 中, $65{ }^{\circ} \mathrm{C}$ 搅拌避光反应, TLC 监测, 反应 $3 \mathrm{~d}$ 后, 产物点 不再发生变化，停止反应。减压蒸干溶剂，剩余物用 $100 \mathrm{~mL} \mathrm{CH} \mathrm{Cl}_{2}$ 溶解, 有机相依次以稀盐酸 $(5 \%)$ 及饱和 $\mathrm{NaCl}$ 洗涤, 无水 $\mathrm{MgSO}_{4}$ 干燥, 过滤. 减压抽干溶剂后粗 产物以柱色谱提纯(石油醚为淋洗液), 得白色固体 $\mathbf{B}_{\mathbf{1}}$, 
$0.830 \mathrm{~g}$, 产率 89\%. m.p. 133.7 $135.4{ }^{\circ} \mathrm{C} ;{ }^{1} \mathrm{H}$ NMR $(400$

$\left.\mathrm{MHz}, \mathrm{CDCl}_{3}\right) \delta: 7.617$ (s, 3H, ArH), $7.567(\mathrm{t}, J=2.8 \mathrm{~Hz}$, $3 \mathrm{H}, \mathrm{ArH}), 7.522$ (t, $J=3.2 \mathrm{~Hz}, 3 \mathrm{H}, \mathrm{ArH}), 7.504$ (t, $J=2.8$ $\mathrm{Hz}, 3 \mathrm{H}, \mathrm{ArH}), 1.986$ (s, 3H, OH), $1.624\left(\mathrm{~s}, 18 \mathrm{H}, \mathrm{CH}_{3}\right)$, $1.136(\mathrm{~s}, 63 \mathrm{H}) ;{ }^{13} \mathrm{C} \mathrm{NMR}\left(100 \mathrm{MHz}, \mathrm{CDCl}_{3}\right) \delta: 135.0$, $134.6,134.4,134.2,124.3,123.7,123,5,123.2,105.0$, 95.1, 92.5, 89.1, 88.6, 80.6, 65.6, 31.4, 18.6, 11.3. Anal. calcd for $\mathrm{C}_{78} \mathrm{H}_{96} \mathrm{Si}_{3} \mathrm{O}_{3}$ : C 80.36, H 8.30; found $\mathrm{C} 80.15, \mathrm{H}$ 8.43 .

\subsubsection{1,3,5-三乙炔基苯 $(\mathbf{1 0})$ 的合成}

向 Schlenk 反应瓶中加入甲苯 $200 \mathrm{~mL}$, 磁力搅拌下 通 $\mathrm{N}_{2} 0.5 \mathrm{~h}$ 后加入 9 (1.32 g, $\left.4 \mathrm{mmol}\right)$. 将溶液升温至 75 ${ }^{\circ} \mathrm{C}$, 加入质量分数为 $40 \%$ 四丁基氢氧化铵的甲醇溶液 (0.77 g, $1.2 \mathrm{mmol})$, 搅拌反应, TLC 监测, $15 \mathrm{~min}$ 后体系 不再变化, 停止反应, 降至室温, 溶液依次用稀盐酸 $(5 \%)$, 饱和 $\mathrm{NaCl}$ 溶液洗涤, 无水 $\mathrm{MgSO}_{4}$ 干燥, 过滤. 减 压蒸干溶剂后粗产物用柱色谱(石油醚为淋洗液)提纯, 得到白色固体 10, $0.55 \mathrm{~g}$, 得率 90\%. m.p. 86.3 87.1 ${ }^{\circ} \mathrm{C}$ (文献值 $\left.{ }^{[28]} 87{ }^{\circ} \mathrm{C}\right) ;{ }^{1} \mathrm{H}$ NMR $\left(400 \mathrm{MHz}, \mathrm{CDCl}_{3}\right) \delta: 7.57$ (s, $3 \mathrm{H}, \mathrm{ArH}), 3.10(\mathrm{~s}, 3 \mathrm{H}) ;{ }^{13} \mathrm{C} \mathrm{NMR}\left(100 \mathrm{MHz}, \mathrm{CDCl}_{3}\right) \delta$ : 135.6, 123.0, 81.6, 78.7.

\section{2 .10 苯乙炔树枝状化合物 $\mathbf{B}_{2}$ 的合成}

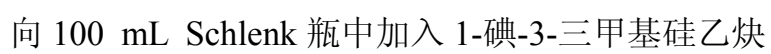
基-5-三异丙基硅乙炔基苯(6) (1.95 g, $4.06 \mathrm{mmol}), \mathrm{CuI}$ (0.023 g, $0.12 \mathrm{mmol}), \mathrm{PdCl}_{2}\left(\mathrm{PPh}_{3}\right)_{2}(0.042 \mathrm{~g}, 0.06 \mathrm{mmol})$, $\mathrm{PPh}_{3}(0.063 \mathrm{~g}, 0.24 \mathrm{mmol})$, 体系抽真空, 通 $\mathrm{Ar}$ 气 4 次后, 用注射器将已鼓泡除氧 $30 \mathrm{~min}$ 的哌啶 $45 \mathrm{~mL}$ 注入瓶中, 然后用注射器向体系滴入已鼓泡除氧 $30 \mathrm{~min}$ 的 $1,3,5$-三 乙炔基苯 $(8)(0.15 \mathrm{~g}, 1.00 \mathrm{mmol})$ 的哌啶溶液 $5 \mathrm{~mL}$, 室温 搅拌避光反应, TLC 监测, 反应 $4 \mathrm{~d}$ 后, 产物点不再发生 变化, 停止反应. 减压蒸干溶剂, 剩余物用 $100 \mathrm{~mL}$ $\mathrm{CH}_{2} \mathrm{Cl}_{2}$ 溶解, 有机相依次以稀盐酸 $(5 \%)$ 及饱和 $\mathrm{NaCl}$ 洗 涤, 无水 $\mathrm{MgSO}_{4}$ 干燥, 过滤. 减压抽干溶剂后粗产物以 柱色谱提纯(石油醚为淋洗液), 得白色固体 $\mathbf{B}_{2}, 0.615 \mathrm{~g}$, 产率 51\%. m.p. 239.1 241.0 ${ }^{\circ} \mathrm{C}$; ${ }^{1} \mathrm{H}$ NMR (400 MHz, $\left.\mathrm{CDCl}_{3}\right) \delta: 7.634$ (s, 3H, ArH), 7.593 (d, $J=1.6 \mathrm{~Hz}, 6 \mathrm{H}$, $\mathrm{ArH}), 7.566$ (t, $J=2.8 \mathrm{~Hz}, 3 \mathrm{H}, \mathrm{ArH}), 1.134$ (s, 63H), 0.256 $(\mathrm{s}, 27 \mathrm{H}) ;{ }^{13} \mathrm{C} \mathrm{NMR}\left(100 \mathrm{MHz}, \mathrm{CDCl}_{3}\right) \delta: 135.1,134.8$, $134.6,134.4,124.2,123.9,123,7,123.2,105.1,103.1$, $95.9,92.4,89.1,88.6,18.6,11.3,-0.2$. Anal. calcd for
$\mathrm{C}_{78} \mathrm{H}_{102} \mathrm{Si}_{6}$ : C 77.54, H 8.51; found C 77.54, H 8.55.

\section{References}

[1] Yang, L.-L.; Dong, Y.-M.; Hu, X.-L.; Liu, A.-H. Acta Chim. Sinica 2012, 70, 21 (in Chinese). (杨柳林, 董炎明, 胡晓兰, 刘安华, 化学学报, 2012, 70, 21.)

[2] Ma, C.; Li, L.; Sun, J.-S.; Zhao, F.; Li, Y.-L. Chin. J. Org. Chem. 2011, 31, 1977 (in Chinese). (马茶, 李龙, 孙金声, 赵丰, 李易隆, 有机化学, 2011, 31, 1977.)

[3] Li, L.; Ma, C.; Sun, J.-S.; Zhao, F.; Pang, Z.-L.; Li, Y.-L. Chin. J. Org. Chem. 2012, 32, 304 (in Chinese).

(李龙, 马茶, 孙金声, 赵丰, 庞正炼, 李易隆, 有机化学, 2012, 32, 304.)

[4] Harigaya, K. Phys. Chem. Chem. Phys. 1999, 1, 1687.

[5] Devadoss, C.; Bharathi, P.; Moore, J. S. J. Am. Chem. Soc. 1996, 118(40), 9635.

[6] Kopelman, R.; Shortreed, M.; Shi, Z.-Y.; Tan, W.; Xu, Z.; Moore, J. S.; Bar-Haim, A.; Klafter, J. Phys. Rev. Lett. 1997, 78, 1239.

[7] Nakano, M.; Fujita, H.; Takahata, M.; Yamaguchi, K. J. Am. Chem. Soc. 2002, 124, 9648.

[8] Wang, P.-W.; Liu, Y.-J.; Devadoss, C.; Bharathi, P.; Moore, J. S. Adv. Mater. 1996, 8, 237.

[9] Wu, Z.; Xiong, Y.; Zou, J.; Wang, L.; Liu, J.; Chen, Q.; Yang, W.; Peng, J.; Cao, Y. Adv. Mater. 2008, 20, 2359.

[10] Astruc, D.; Boisselier, E.; Ornelas, C. Chem. Rev. 2010, $110,1857$.

[11] Ayabe, M.; Ikeda, A.; Kubo, Y.; Takeuchi, M.; Shinkai, S. Angew. Chem., Int. Ed. 2002, 41, 2790.

[12] Xu, Z.-F.; Moore, J. S. Polym. Prep. 1993, 1, 128.

[13] Adhikari, R. M.; Duan, L.; Hou, L.-D. Chem. Mater. 2009, 21, 4638.

[14] Rana, D.; Gangopadhyay, G. Chem. Phys. Lett. 2001, 334, 314.

[15] Li, J.; Huang, P.-C. Res. Chem. Intermed. 2012, 38, 403.

[16] Pesak, D. Ph.D. Dissertation, University of Illinois, Illinois, 1995.

[17] Chen, Q.-Y.; Ma, Y.-W.; Wang, C.-Y.; Shen, Y.-J. Chin. J. Org. Chem. 2012, 32, 1526 (in Chinese). (陈秋燕, 麻玉雯, 王成云, 沈永嘉, 有机化学, 2012, 32, 1526.)

[18] Littke, A. F.; Fu, G.-C. Angew. Chem., Int. Ed. 2002, 41, 4176.

[19] Siemsen, P.; Livingston, R. C.; Diederich, F. Angew. Chem., Int. Ed. 2000, 39, 2632.

[20] Ji, Z.; Li, S.; Li, Y. Inorg. Chem. 2010, 49, 1337.

[21] Melissaris, A. P.; Litt, M. H. J. Org. Chem. 1994, 59, 5818.

[22] Li, J.; Huang, P.-C. Beilstein J. Org. Chem. 2011, 7, 426.

[23] Lim, Y.-K.; Jiang, X.; Bollinger, J. C.; Lee, D. J. Mater. Chem. 2007, 17, 1969.

[24] Baxter, P. N. W. Chem. Eur. J. 2003, 9, 5011.

[25] Xu, Z.; Moore, J. S. Angew. Chem., Int. Ed. 1993, 32, 246.

[26] Rodriguez, J. G.; Esquivias, J.; Lafuente, A.; Diaz, C. J. Org. Chem. 2003, 68, 8120 .

[27] Bo, Z.-S.; Schluter, A. D. J. Org. Chem. 2002, 67, 5327.

[28] Yoosaf, K.; Llanes-Pallas, A.; Marangoni, T.; Belbakra, A.; Marega, R.; Botek, E.; Champagne, B.; Bonifazi, D.; Armaroli, N. Chem. Eur. J. 2011, 17, 3262. 